\title{
Las villas de Luigi Manini en Sintra (1890-1912)
}

\author{
Iván MOURE PAZOS \\ Istituto di Studi Superiori - Università di Bologna \\ CIAUD - Faculdade de Arquitectura - Universidade de Lisboa \\ Departamento de Historia da Arte - Universidade de Santiago de \\ Compostela \\ ivan.menes@yahoo.es
}

Recibido: $15 / 12 / 2013$

Modificado: $21 / 10 / 2014$

Aceptado: $21 / 11 / 2014$

\section{Resumen}

El presente artículo trata de ser un recorrido por los principales logros arquitectónico-paisajísticos alcanzados por Luigi Manini en la Sierra de Sintra, a través de cuatro de sus obras más aclamadas: Casa del Giardiniere-Biester, Villa Sassetti, Chalet Lima Mayer y Quinta da Regaleira.

Palabras clave: Sintra, Luigi Manini, Casa del Giardiniere-Biester, Villa Sassetti, Chalet Lima Mayer, Quinta da Regaleira.

Title: Luigi Manini's villas at Sintra (1890-1912)

\section{Abstract}

This article tries to be a tour of the main architectural-landscape achievements of Luigi Manini in Sintra, through his most acclaimed works: Casa del Giardiniere-Biester, Villa Sassetti, Chalet Lima Mayer, and Quinta da Regaleira.

Keywords: Sintra, Luigi Manini, Casa del Giardiniere-Biester, Villa Sassetti, Chalet Lima Mayer, Quinta da Regaleira.

\section{Índice}

1. Introducción

2. El Chalet Biester y la introducción de tipologías centroeuropeas

3. Villa Sassetti y la búsqueda del genius loci

4. Chalet Lima Mayer o cuando la escenografía deviene en arquitectura

5. La Quinta da Regaleira y la exaltación del "anima lusitana"

6. Conclusión 


\section{Introducción}

Parece imposible escribir sobre Sintra sin sucumbir al apasionamiento que provoca su simple evocación ${ }^{1}$. Capital del Romanticismo europeo, ha sido la musa de innúmeros poetas, artistas, músicos, y sensibles arquitectos que no dudaron en inmortalizar, en sus diferentes producciones artísticas, su atmósfera onírica y fantasmática. Almeida Garret, Eça de Queirós, William Beckford, Lord Byron, Robert Southey, Hans Christian Andersen, Richard Strauss, Fernando Pessoa, Aleister Crowley o Luigi Manini son algunas de las variopintas personalidades que, indefectiblemente, amalgaman la identidad sintrense.

Las villas de la Sierra -más propiamente llamadas Quintas- se erigen como un excelente testimonio de la ostentosidad pretérita de la Sintra decimonónica. Ocultas bajo un boscoso manto de frondosidad vegetal -que cabe adjetivar de pleistocena- ofrecen al visitante una de las "escenografías" arquitectónicas más exuberantes del planeta. Y es que resulta difícil encontrar en el orbe terrestre un elenco de villas que regalen a la vista tal recreación de lujo y fantasía. La gran mayoría obedece a un gusto estético puramente romántico, pero éstas no pueden ser catalogadas en base a criterios estilísticos puramente arquitectónicos. Cierto es que, en muchas de estas edificaciones, asoman con insistencia diferentes estilos decimonónicos (neomanuelino, neoislámico, neorrománico...), pero de ningún modo obedecen a unas pautas ortodoxas puramente definibles. Por lo tanto, debemos considerar que en dichas creaciones prima, ante todo, el capricho y la libertad tectónica, supeditada, en muchas ocasiones, a programas iconográficos complejísimos así como a la búsqueda de un adecuado genius loci que las ensalza entre la espesura de un paisaje organicista. Esta idiosincrasia infiere al lugar una riqueza artística sin parangón, donde extravagantes planteamientos arquitectónicos -fuertemente individualizadosemergen de una espesura natural imponente, onírica y gigantizada.

Uno de los artífices fundamentales que posibilitaron la creación de este delirio "teatral" fue el arquitecto, escenógrafo, decorador y pintor italiano Luigi Manini (1848-1936) que, en 1879, se desplaza a Portugal para trabajar -durante más de treinta años- en diferentes proyectos artísticos, antes de su regreso definitivo a tierras

${ }^{1}$ El presente artículo forma parte del proyecto internacional "Laboratorio di Ricerca sulle Città" (Istituto di Studi Superiori, Università di Bologna). Asimismo, también del proyecto europeo "Performigrations: people are the territory" (Dipartimento di Lingue, Letterature e Culture Moderne, Università di Bologna). Análogamente, dicho artículo ha sido posible gracias a la financiación postdoctoral del Plan Galego de Investigación, Innovación e Crecemento 2011-2015 promovido por la Xunta de Galicia. Gran parte de la bibliografía de este artículo se encuentra editada únicamente en italiano, inglés y portugués. Todos los textos citados han sido traducidos por el autor al castellano. 
lombardas en 1912 (D'Amia 2007: 53-63). Durante este período, se convertirá, por la potencia escenográfica de sus proyectos y sus inagotables recursos artísticos, en el artista más solícito de todo Portugal y de Sintra en particular ${ }^{2}$. Sólo en la sierra sintrense, nos ha legado cuatro obras maestras de la arquitectura finisecular. Cuatro joyas que, cabe decirlo, constituyen -junto al colosal Palacio de Buçaco (1898-1912)- el mejor corolario de su carrera como creador. Se trata de su obra más aclamada, compuesta por su Casa del Giardiniere-Biester y el programa decorativo de la casa (1890-1891), Villa Sassetti (1890), Villa Lima Mayer (1897) y Quinta da Regaleira (1898-1912).

Lo que sigue, pretende poner en valor dichas obras, aquilatadas por la pátina del tiempo. Cada una de ellas representa una tipología arquitectónica diferente $y$ ha sido concebida de manera particularizada, convirtiéndose en auténticos iconos de la arquitectura maniniana. La introducción de cuadros tipológicos centroeuropeos (Biester), la búsqueda de un genius loci y el óptimo aprovechamiento de la potencialidad del lugar (Sassetti), la escenografía teatral convertida en arquitectura (Mayer), así como el gusto por la reminiscencia arqueológica y nacional (Regaleira), son, actualmente, señas identitarias de Luigi Manini y, por extensión, de la ciudad de Sintra. Es preciso, por lo tanto, adentrarse en la espesura de la Sierra para encontrarnos con el verdadero genio de Luigi Manini.

\section{El Chalet Biester y la introducción de tipologías centroeuropeas}

En 1890, el rico empresario alemán Frederico Biester ${ }^{3}$ encarga al arquitecto José Luis Monteiro la construcción de una villa al estilo inglés Queen Anne (fusión de neogótico y neorrománico) (Cunha 2004: 59), de cuyo interior -concretamente de los frescos pictóricoshabrá de encargarse Luigi Manini en feliz connubio con uno de los más grandes ebanistas de la época, Leandro Braga (Alpini 2007: 117129). Este diálogo fructificará en un trabajo conjunto difícilmente parangonable ${ }^{4}$. No sin razón, Roman Polansky optó por situar parte de la acción de su The Ninth Gate (1999) en los interiores de este complejísimo cuadro espacial.

\footnotetext{
2 Sobre el contexto biográfico-artístico de Manini, vid. Pereira y Luckhurst (2006a: 11-19).

3 Durante años, se mantuvo la hipótesis de que dicha obra había sido promovida por el dramaturgo lisboeta de apellido homónimo, pero, actualmente, esta tesis se considera errónea por un simple desacuerdo cronológico, pues el Chalet Biester se encargará en 1890, diez años después de la muerte del escenógrafo.

${ }^{4}$ La Villa pasó, el 6 de diciembre de 1995, a engrosar la lista de monumentos Patrimonio de la Humanidad de la UNESCO.
} 
En los jardines exteriores, Manini colaboró con el célebre paisajista francés François Nogré, proyectando una de las obras más importantes del Portugal fin de siècle, la llamada Casa del Giardiniere-Biester (1891) (Pereira y Luckhurst 2007: 76). "De esta colaboración entre Manini y Nogré surgirá el proyecto para la casa y el jardín de Luis Canedo en Vila da Feira (1904)" (Luckhurst y Pereira 2007: 91), con lo cual, nos encontramos ante una obra inicial que asienta una importante tipología edilicia, como bien subraya la especialista Gaia Piccarolo en su genial artículo "Villa e Villino: il tema dell'abitazione Borghese nell'opera di Luigi Manini" (Piccarolo 2007: 101). Sabemos, por Gerald Luckhurst y Denise Pereira, que "en el proyecto Biester el contacto de Manini con Nogré fue cotidiano" (Luckhurst y Pereira 2007: 91). Partiendo de este hecho, no ha de extrañarnos que, trece años después, ambos retomen un proyecto conjunto de iguales características. Un simple rastreo a vuelapluma, evidencia las similitudes entre sendos proyectos concebidos diacrónicamente al modo centroeuropeo del Chalet Suizo.

Pero la importancia de la Casa del Giardiniere-Biester va más allá de haberse convertido en un mero genotipo arquitectónico o plantilla constructiva para obras futuras. Cabe recordar que nos encontramos ante la primera creación de estas características aparecida en el nutrido repertorio arquitectónico portugués, incluso precediendo a la célebre Villa Sassetti en Estoril (1892) proyectada por su homólogo italiano Sebastiano Guiseppe Locati (Ferreira 2007: 65). La Casa del Giardiniere-Biester constituye un ejemplo palmario de la nueva concepción ochocentista de vivienda, elevando a rango de villa burguesa la vivienda del trabajador y su familia, adhiriéndose y equiparándose ésta a la casa del propietario creada por Monteiro (Piccarolo 2007: 101). La relación de esta querencia maniniana por la tipología arquitectónica alpina ha sido estudiada ampliamente por Gaia Piccarolo: "El referimiento al Chalet Suizo es recurrente en los materiales del fondo Manini, conservados en el Museo Cívico de Crema y del Cremasco" (Piccarolo 2007: 100). Y es también la autora quien ha tenido la astucia de relacionar la Casa del GiardiniereBiester con una de las estampas centroeuropeas aparecidas en el fondo documental de Manini en Crema y que reproduce, de manera fehaciente -por no decir clónica-, el proyecto del arquitecto para Sintra. Se trata de la Villa Wollank (1874-1875), erigida por Hugo Georg Licht en Berlín quince años antes del proyecto para Biester (Piccarolo 2007: 106).

Por lo tanto, y a tenor de lo anteriormente expuesto, no será disparatado establecer un claro vínculo inspirador entre estas cuatro arquitecturas interconectadas: Villa Wollank, Casa del GiardiniereBiester, Villa Sassetti y Casa y Jardín de Luis Canedo. Todas ellas, ejemplos palmarios del fasto burgués finisecular y hoy ya piezas de interés turístico internacional (figuras 1 a 4 ). 


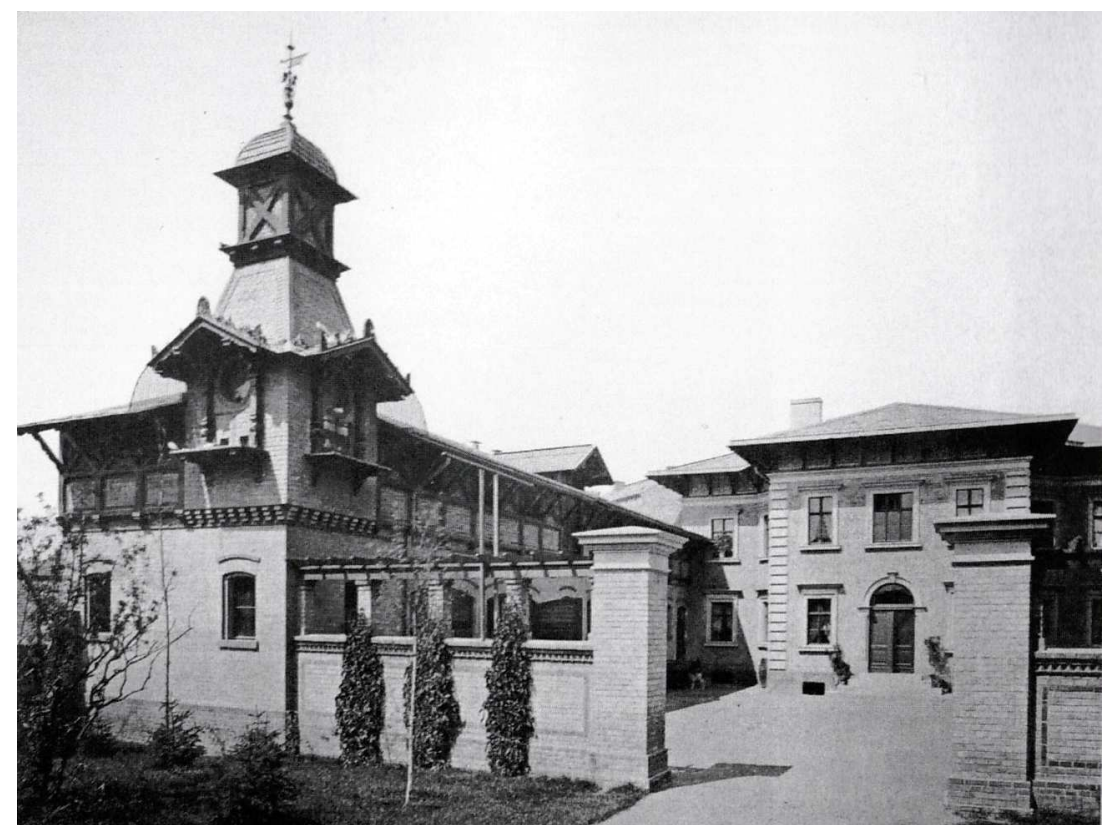

Figura 1. Hugo Licht, Villa Wollank en Berlín, 1874-1875. Museo Cívico de Crema y del Cremasco (Crema).

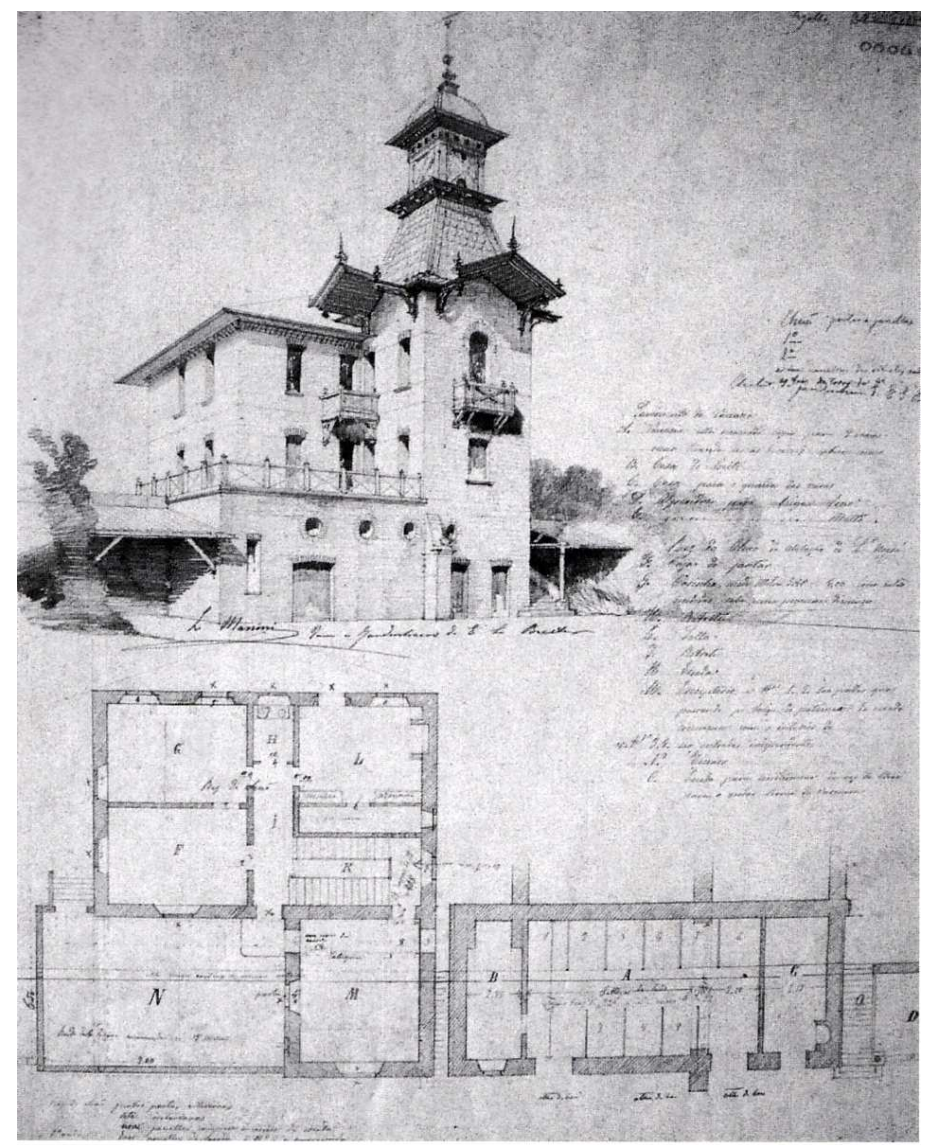

Figura 2. Luigi Manini, Casa del Giardiniere Biester, 1891. Museo Cívico de Crema y del Cremasco (Crema). 


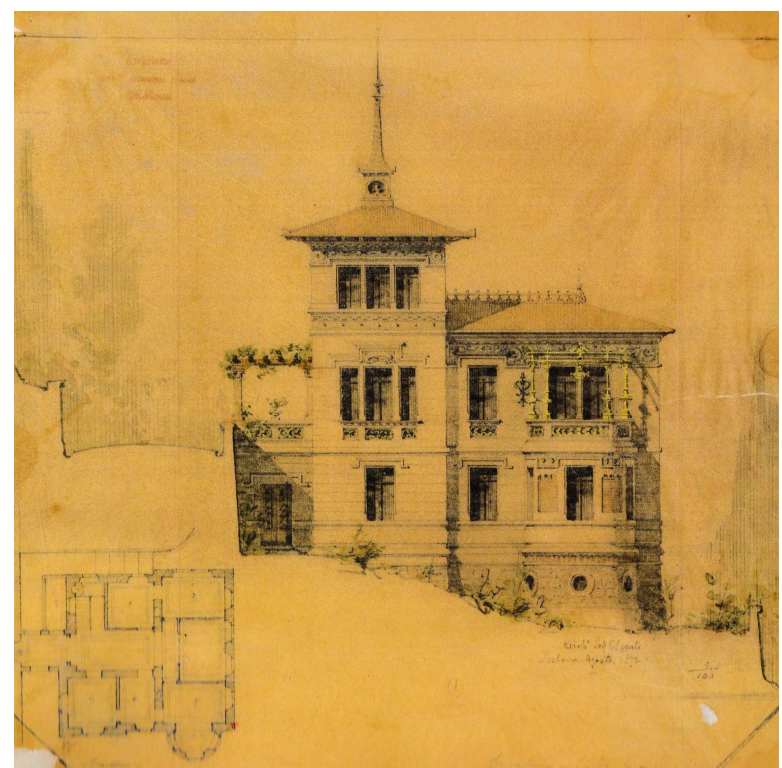

Figura 3. Sebastiano Giuseppe Locati, Villa Sasetti en Estoril, 1892. Departamento de Ingeniería Constructora y del Territorio, Universidad de Pavía (Pavía).

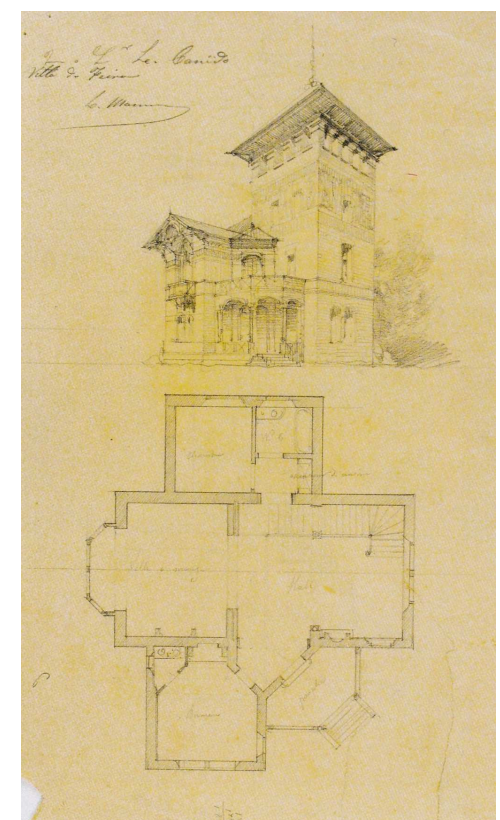

Figura 4. Luigi Manini, Casa de Luis Canedo en Vila da Feira, 1904-1905. Museo Cívico de Crema y del Cremasco (Crema). 


\section{Villa Sassetti y la búsqueda del genius loci}

Dos años antes de que Sebastiano Guiseppe Locati llevara a cabo su citado proyecto de Villa Sassetti en Estoril, Luigi Manini diseña para su amigo Victor Carlos Sassetti una auténtica genialidad de inspiración neolombarda en la Sierra de Sintra (Roncai 2007: 39-45) (figura 5). Se trata de su primera obra arquitectónica en la ciudad portuguesa, precediendo en un año al proyecto arquitectónico para Biester (Pereira y Luckhurst 2004: 253-280, 2008: 200-210). La Villa Sasseti (1890) -de reciente adquisición y remodelación por parte del gobierno portugués- es una importante joya arquitectónica, pero también una de las grandes proezas escenográficas maninianas. No debemos olvidar que Luigi Manini era, ante todo, escenógrafo y que carecía de formación como arquitecto. Incluso, pudiera llegar a argumentarse que su vocación arquitectónica se produjo de una manera un tanto azarosa y aleatoria (Piccarolo 2005: 272-276). "El paso de la arquitectura a la escenografía fue gradual", por no decir accidentada, por una serie de hechos fortuitos que le propiciaron grandes simpatías entre la burguesía del momento (Pereira y Luckhurst 2007: 73, 75). De lo que se deduce que gran parte de su éxito como arquitecto está empapado de su pasado de escenógrafo. Redundar en que la arquitectura de Manini resulta altamente escenográfica se antoja una evidencia incuestionable. La Villa Sassetti constituye un ejemplo claro de este juego de perspectivas teatrales. Desde cualquiera de los grandes monumentos sintrenses exceptuando el Palacio de Montserrate (1858)-: Paço da Vila (1489), Castelo dos Mouros (siglo VIII), Chalet Biester, Palacio da Pena (1836) y Quinta da Regaleira, se divisa, imponente, la Villa Sassetti (figura 6). Pudiéramos atribuir este hecho al azar, pero lo cierto es que resulta poco factible -a tenor de las distancias existentes entre los monumentos y la escasa visibilidad que ofrece la frondosidad vegetal de la Sierra- que tal alineamiento sea una coincidencia; máxime sabiendo que en las restantes creaciones de Manini esto se revela como un leitmotiv recurrente. De tal modo que se establece una correlación escenográfica entre los principales monumentos, a través de un interesante juego de perspectivas; de un verdadero genius loci global, inmortalizado en las fotografías que el autor, en sus frecuentes paseos por la sierra, nos ha legado (Piccarolo 2005: 276-307):

La búsqueda de los mejores ángulos, de las perspectivas más favorables, le llevaron a caminar por la montaña, aspecto que se evidencia en las fotos que quitó de Sintra. Dichas fotos, tienen el poder de revelar la imaginación de Manini. Detrás de la máquina fotográfica, se coloca en la posición de observador, de localizador, buscando hacer suyo el espíritu del lugar [...] reflejado en sus intervenciones paisajísticas de Sintra $[. .$.$] , existiendo un perfecto$ 
paralelismo entre el constructor de paisajes y el inventor de la escena. Este universo (pintura y arquitectura) se entrelazan en su proceso creativo, patentizando el hecho de que la observación de la naturaleza y la narrativa lírica se transforman en los principales soportes de la conceptualización del artista [...]. Esta afirmación se hace evidente en la colina de Sintra. Los edificios de Manini fueron concebidos como elementos constituyentes y generadores de nuevos paisajes. Subrayando el aura del Palacio da Pena, del Castelo dos Mouros, del Palacio Real, sus proyectos recrean el paisaje sintrense como si fuese irreal [...]. (Luckhurst y Pereira 2007: 90)

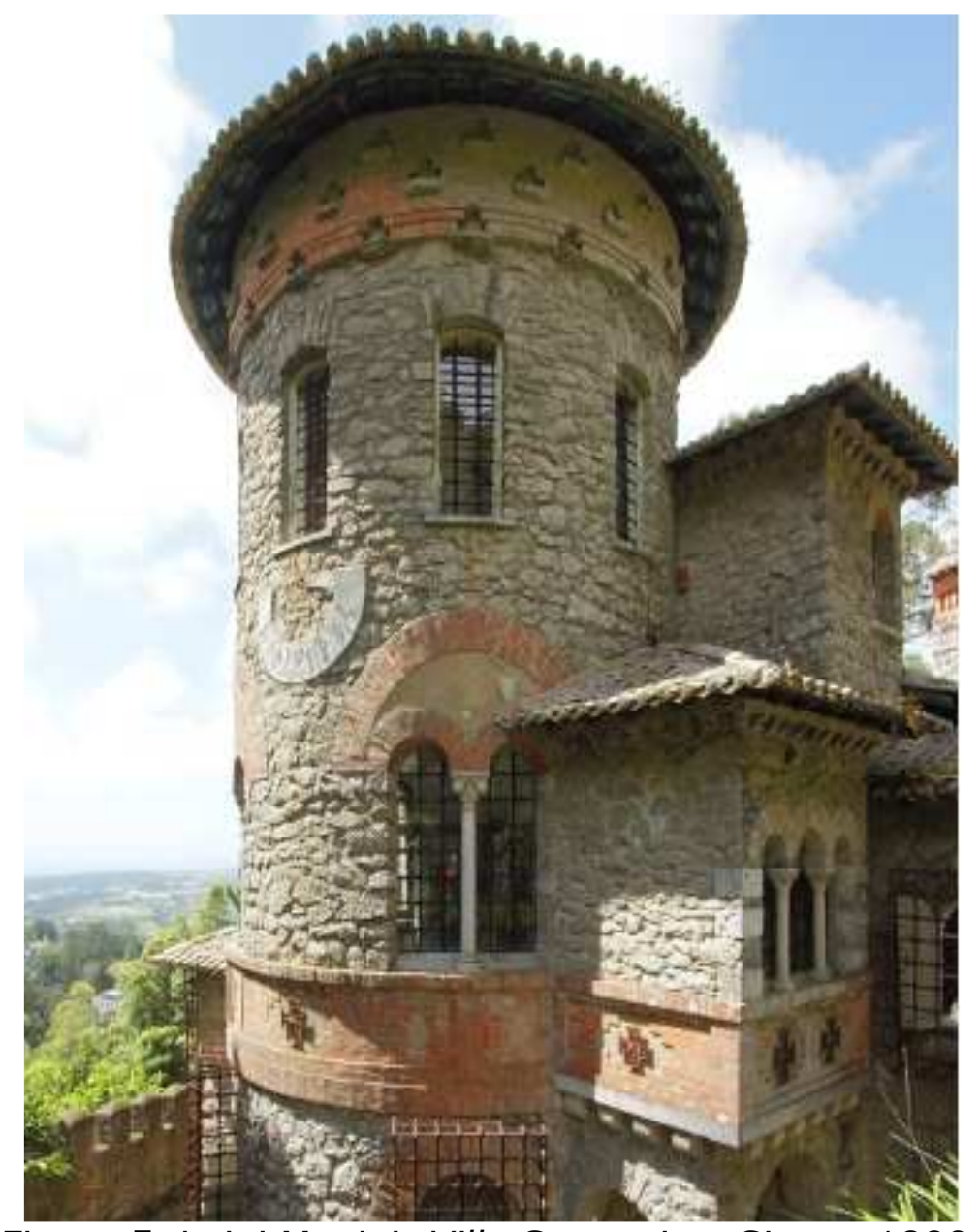

Figura 5. Luigi Manini, Villa Sassetti en Sintra, 1890. 


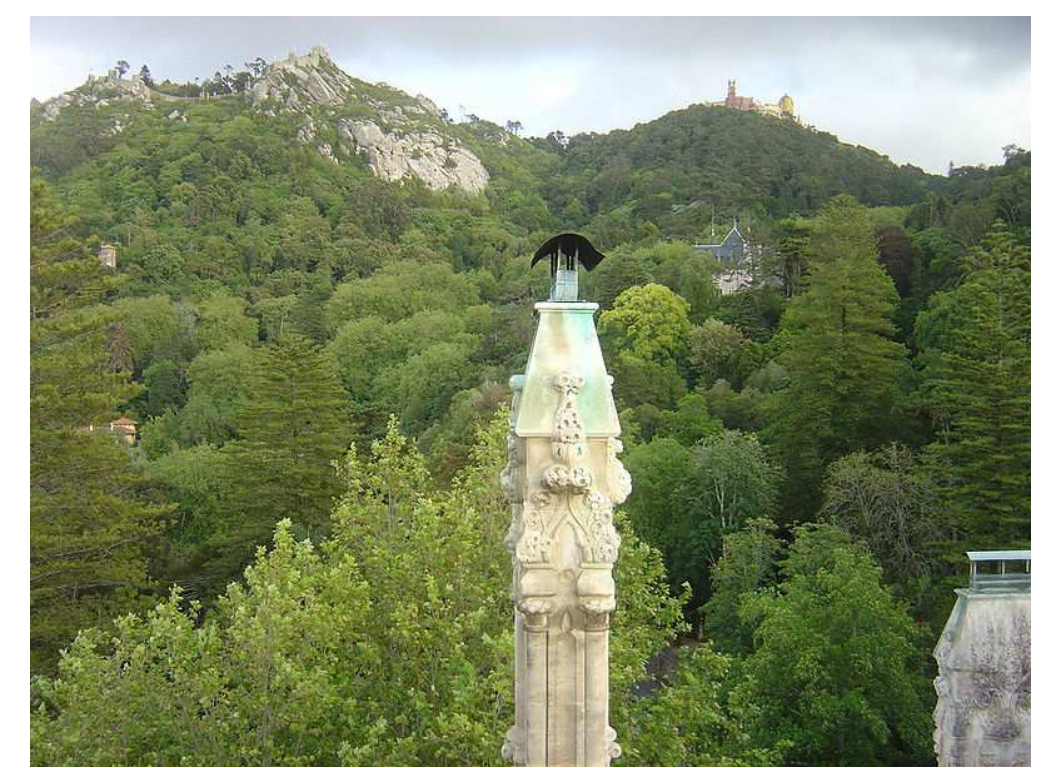

Figura 6. Foto panorámica tomada desde la torre de la Quinta da Regaleira en Sintra. De izquierda a derecha, Villa Sassetti, Castelo dos Mouros, Palacio da Pena y, de frente, el Chalet Biester.

No debe obviarse que Manini fue el arquitecto más prolífico de Sintra y, como tal, concibió la ciudad como una verdadera "mesa de operaciones" donde poder ejecutar sus creaciones a su antojo. En Villa Sassetti, esta potencia escenográfica se percibe de manera clara y evidente. Con razón apostilla Gaia Piccarolo:

La Villa Sassetti definida por Manini como "construcción de estilo lombardo", es un pequeño e interesante ensayo de arquitectura castellana del norte de Italia filtrado a través del gusto romántico de lo pintoresco de derivación inglesa, atendiendo a la integración de la arquitectura en el paisaje y al máximo aprovechamiento de la potencialidad del lugar. La posición privilegiada de su altura, y la adaptabilidad de su planimetría irregular, la libre yuxtaposición de volúmenes y la valorización de la diferencia de alturas hacen que el edificio parezca siempre distinto según el punto de vista, traduciendo al exterior su función interna y permitiendo tener una mayor superficie de ventilación, requisito fundamental para las exigencias de salubridad propias del momento. (Piccarolo 2007: 100) 


\section{Chalet Lima Mayer o cuando la escenografía deviene en arquitectura}

Bien conocidas fueron las amistades femeninas de Luigi Manini en Sintra. Principalmente, se trataba de mujeres pertenecientes a la alta burguesía con las que el autor mantenía un trato cordial, próximo e intelectual. Entre éstas se contaban Amelia Chamiço Biester, Patrocinía de Barros Lima, Perpétua Monteiro, O’Neill y, por supuesto, Lima Mayer, quien le encarga el proyecto de su residencia en la sierra. Dicho proyecto se revela crucial a la hora de exponer una de las características principales de la obra de Manini: la reutilización y conversión de pretéritos proyectos escenográficos en arquitectura, convirtiendo sus obras bidimensionales en realizables estructuras tridimensionales ${ }^{5}$.

Gaia Piccarolo, en su obra Luigi Manini (1848-1936). L'opera architettonica di uno scenografo italiano in Portogallo (2005), ha sido la primera en establecer un vínculo claro entre el pasado escenográfico de Manini y su posterior desarrollo como arquitecto, llegando a considerar la Villa Mayer como un proyecto de "alta inspiración escenográfica" (Piccarolo 2005: 101) y claro ejemplo de esta continua dialogicidad. Dicho diálogo va más allá de lo meramente paisajístico. No se trata solo de una influencia implícita (la búsqueda de un genius loci supeditado a la orografía del terreno y la optimización de perspectivas visuales, anteriormente abordadas), sino que ésta se explicita en los propios proyectos arquitectónicos del artista. De tal modo que muchas de las obras delineadas ya estaban contenidas en precedentes proyectos operísticos y teatrales, al modo de plantillas constructivas: "[...] los elementos que componen los edificios [...] son los mismos usados en la arquitectura fantástica que puebla su escenografía" (Piccarolo 2005: 270). En este sentido, y de manera indudable, el edificio torreado del complejo Lima Mayer puede ser concebido como la "consumación" de su obra escenográfica precedente para el Tannhäuser (1891-1892) de Richard Wagner, del mismo modo que "la escena para Bailado Casati, o para La Favorita" representan gran parte de "los edificios del Palacio O'Neill, Marqués de Foz o el Palacio Cabral [...]"; así como "el castillo sobre el mar de Mefistófeles" evoca, de manera reincidente, el Palacio O'Neill levantado a pie de playa en Cascais (Piccarolo 2005: 270).

En el caso Lima Mayer, baste una mirada rápida para advertir que tal proyección emulsiona como un genotipo del Tannhäuser realizado cinco años antes: en los elementos torreados, los chapiteles, la orografía del lugar... (figuras 7 y 8 ).

5 Sobre esta relación arquitectónico-escenográfica de Manini, vid. Pereira (2012: 147-159) y Pereira y Luckhurst (2006b: 21-63). 

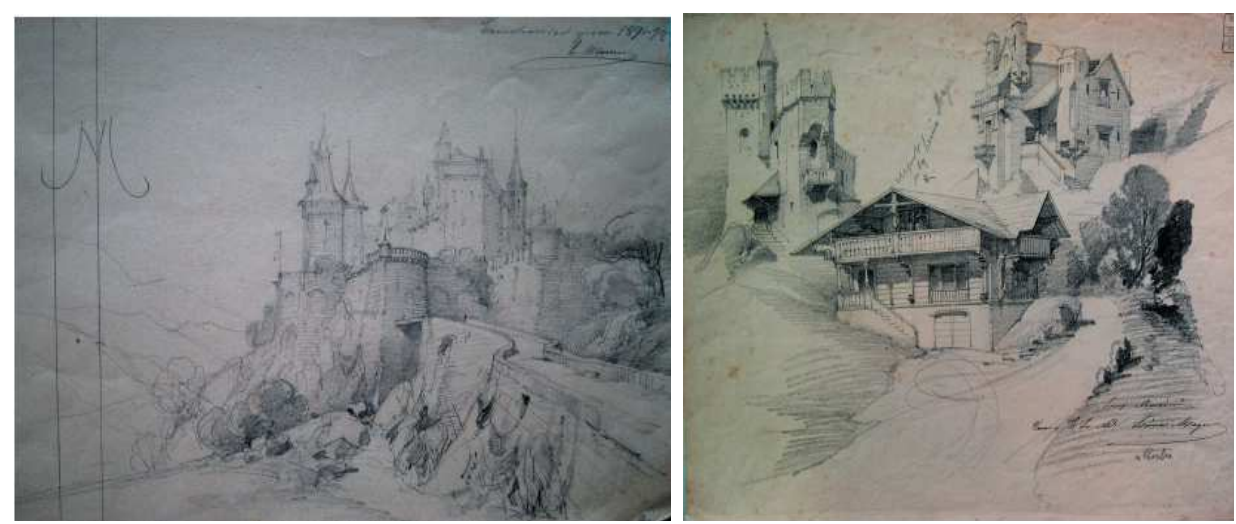

Figuras 7 y 8 . Luigi Manini, Boceto para una escena de Tannhäuser de Richard Wagner, 1891-1892. Teatro Sao Carlos, Museo del Teatro (Lisboa); y proyecto de edificios para Villa Lima Mayer en Sintra, 1897. Museo Cívico de Crema y del Cremasco (Crema).

Este paso de lo escenográfico a lo arquitectónico ha sido bien explicado por Gerald Luckhurst y Denise Pereira, al atribuirle a Manini una cierta frustración inicial como creador. Relegado, inicialmente, al papel de decorador escenográfico, no se le brindó la oportunidad de crear proyectos ad hoc individualizados. Esto le llevaría, andando el tiempo, y tan pronto tuvo oportunidad, a materializarlos a través de la arquitectura pintoresca de la Sierra de Sintra y del Chalet Lima Mayer:

La capacidad de inventar la escena era casi exclusiva del escenógrafo titular y no solo del escenógrafo pintor al que nos referimos. En Portugal, por circunstancias coyunturales económicas, y por la resistencia del público a la introducción del naturalismo y el realismo en la escena nacional, la oportunidad de Manini de ejercitar esta cualidad de inventor de escenas en los espectáculos fue poco frecuente. En este sentido, en Sintra, se impone como arquitecto y paisajista, con innegable capacidad y creatividad. (Luckhurst y Pereira 2007: 96)

\section{La Quinta da Regaleira y la exaltación del "anima lusitana"}

Pocos artistas han tenido la suerte de poder llevar a cabo un proyecto de tal magnitud como la Quinta da Regaleira en Sintra (Malcolm 2003: 198-201) (figura 9). Se trata de la obra más importante de Manini: grutas subterráneas, acuarios, lagos, pozos iniciáticos, trufados de mil y un delirios pintorescos, se abren camino por senderos laberínticos, donde la realidad y la ficción se entreveran (Attlee 2007: 161). Estamos ante una obra a todas luces excesiva e inabordable tanto por sus dimensiones como por su complejidad iconográfica, de corte, como veremos, fuertemente camoniano. Al igual que en el ya realizado Palacio de Buçaco en Coimbra, Manini opta por erigir un palacio de fuertes influencias neomanuelinas, bajo 
el encargo y la cercana supervisión de su benefactor, Augusto Carvalho Monteiro:

Actualmente, es un hecho constatado que el aspecto mágico y taumatúrgico de la arquitectura del parque de la Regaleira deba su audaz programa A Carvalho Monteiro. [...] era natural para Monteiro señalar a Manini una vista o un detalle escenográfico que ilustrase su idea para la Regaleira, cosa que facilitaba el diálogo entre los dos. (Luckhurst y Pereira 2007: 92)

Se trata del segundo proyecto encargado por Monteiro para Sintra, tras verse frustrado el proyecto inicial de Henri Lusseau debido a que éste "no sabía interpretar el estilo neomanuelino requerido por el propietario" (Luckhurst y Pereira 2007: 92) (figura 10). Resulta evidente que la interpretación historicista de este primer proyecto obedecía a un gusto típicamente francés no grato al programa previamente delineado por Monteiro. Sabemos que éste fue un gran coleccionista y bibliófilo empedernido, y que su programa iconográfico para el parque -basado enteramente en la obra de Camões- se impuso como condición sine qua non desde el planteamiento inicial. Esto, en cierto modo, explicaría la falta de éxito del primer proyecto de Lusseau, de tendencia puramente parisina $y$, en consecuencia, alejado de la pretendida querencia lusa estipulada por Monteiro.

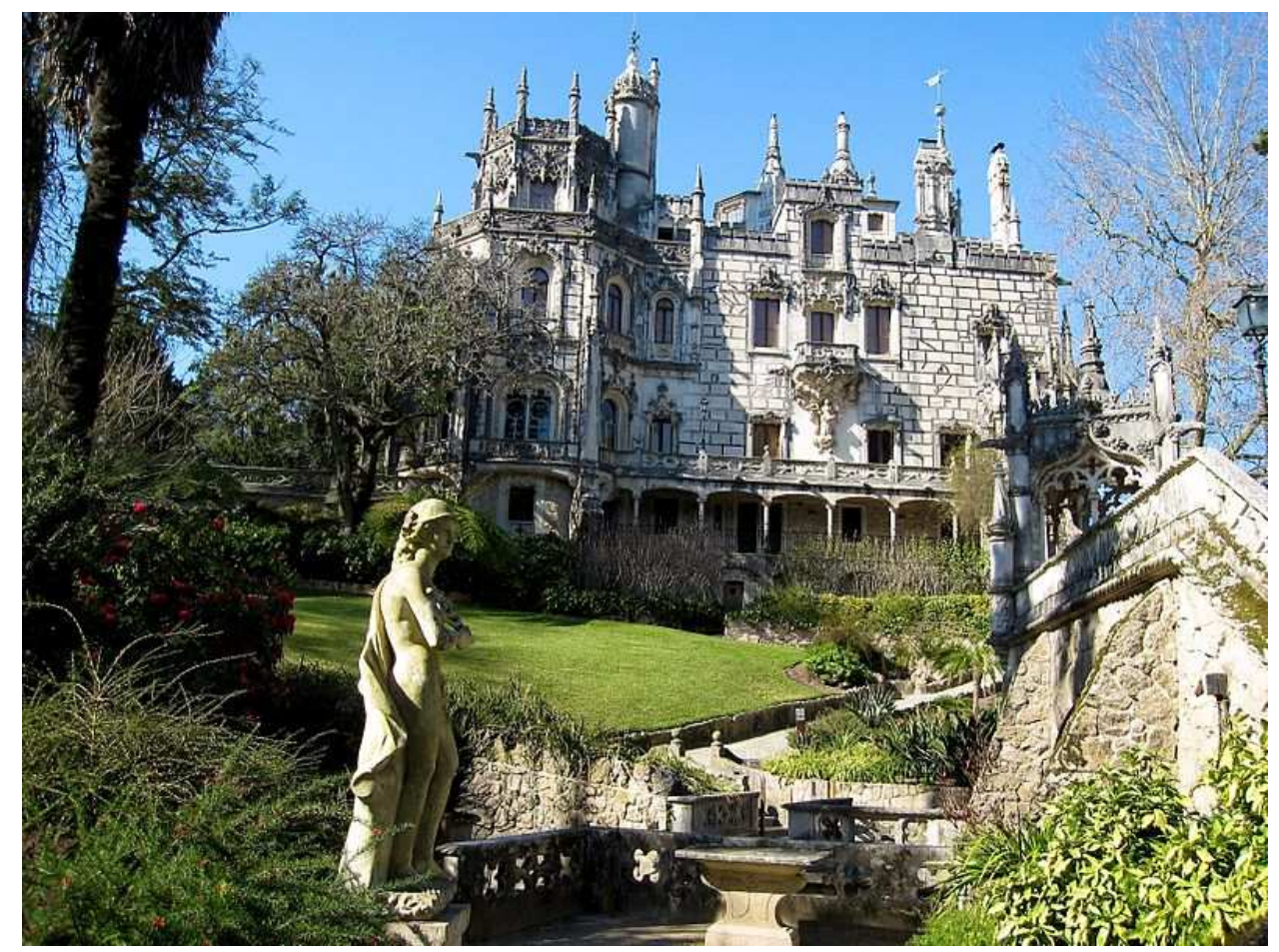

Figura 9. Luigi Manini, Quinta da Regaleira en Sintra, 1898-1912. 


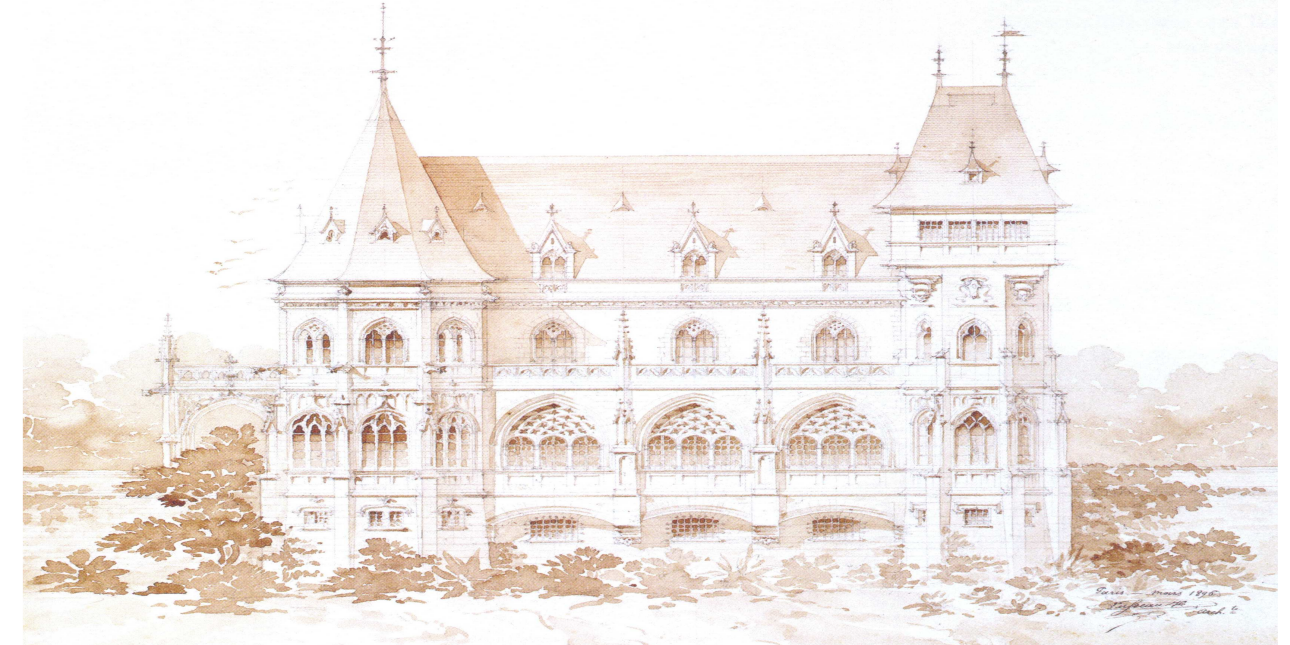

Figura 10. Henri Lusseau, primer proyecto para Quinta da Regaleira, 1896. Cámara Municipal de Sintra, Archivo histórico (Sintra).

Por entonces, Manini ya había realizado el Palacio de Buçaco en Coimbra reinterpretando el estilo manuelino, lo cual ofrecía a Monteiro una auténtica garantía para su pretendida exaltación lusitana (Luckhurst y Pereira 2004: 51-63; Denby 1998: 248) (figura 11). Hemos de tener en cuenta que, en las postrimerías del XIX, los estilos autóctonos en Sintra se veían relegados a antiguos edificios institucionales como el Paço da Vila (1489), ya que pocos optaban por el historicismo portugués para erigir sus villas fin de siècle en la sierra. Por estas fechas, Sintra ofrecía un excelente muestrario de tipologías arquitectónicas que parecían desdeñar su propia identidad portuguesa. Palacio da Pena (Alemania), Chalet Biester (Inglaterra), Villa Sassetti (Italia), Palacio de Montserrat, Quinta do Relogio (Marruecos), fueron sintomáticas de este empeño. La construcción de la Quinta da Regaleira supuso la introducción de añejos postulados neomanuelinos en las grandes villas sintrenses del siglo XIX. No debemos olvidar que, por entonces, el fervor nacionalista portugués vino precedido por una serie de circunstancias sociopolíticas - pérdida de las colonias, innúmeros problemas políticos- que redundaron en una fuerte crisis económica que propició la exaltación de la llamada "ánima lusitana", reivindicando la gran época dorada y expansionista de Don Manuel I (1491-1521) (D'Amia 2007: 45-49). La Quinta da Regaleira, todavía hoy, constituye el mejor ejemplo de este sentimiento portugués proyectado por Manini. A través de "pócimas" historicistas, que él mismo denominaba como "verdad histórica" nutrida a partes iguales de escenografía teatral y gusto arqueológicose erigió como el arquitecto más célebre de la sierra sintrense. La Quinta da Regaleira es, actualmente, su mejor obra; una obra de arte total que nos remite a los fastos, ya extintos, de aquel manuelino 
pretérito, trufado, en filigrana, de las licencias interpretativas del genio italiano.

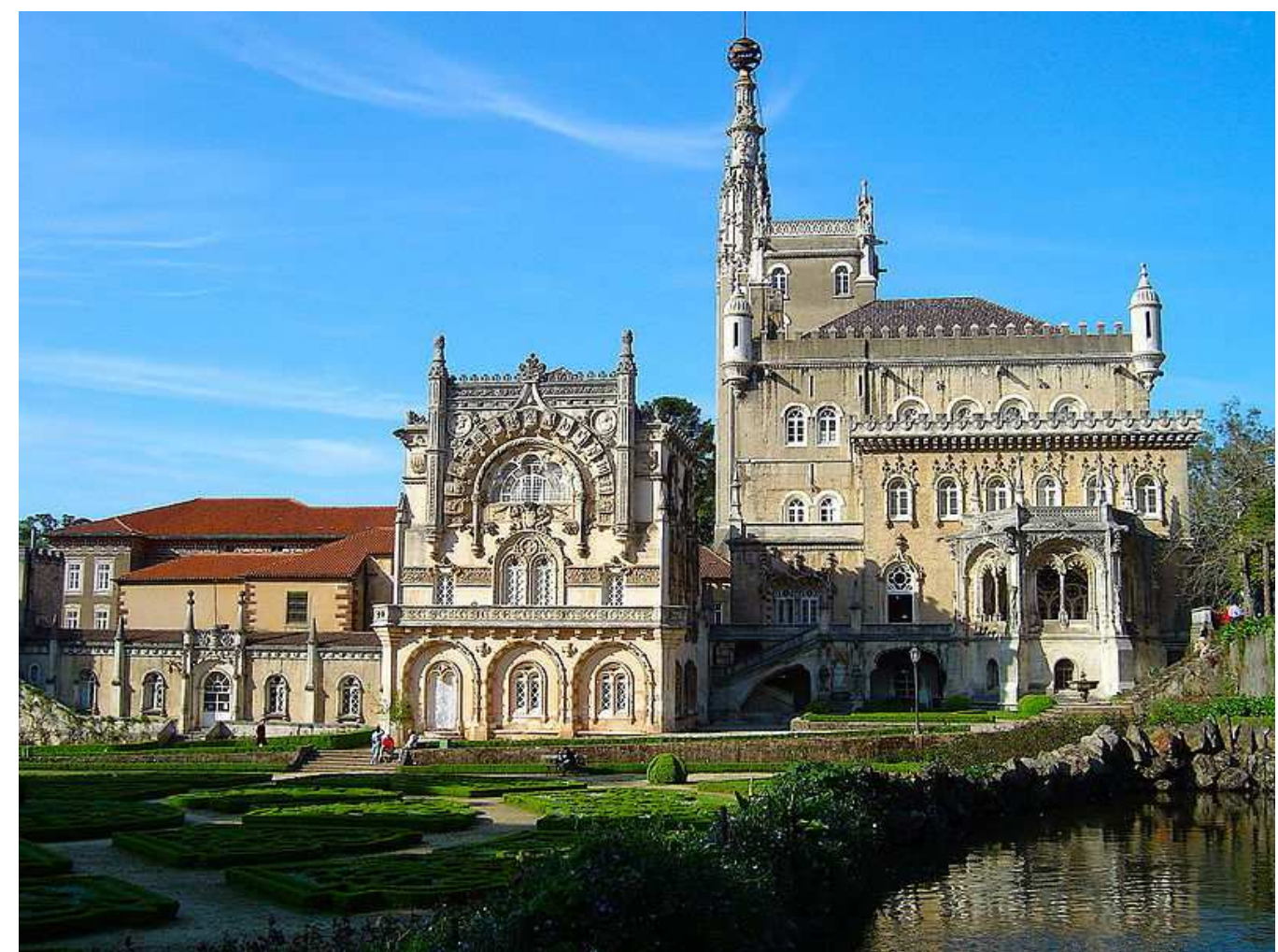

Figura 11. Luigi Manini, Palaço de Buçaco en Coimbra, 1888-1907.

\section{Conclusión}

Después de este breve recorrido por las villas de Manini, parece incuestionable constatar su enorme importancia en la sierra sintrense como creador fundamental -que no fundacional- de la capital del romanticismo europeo. Sintra ha sido el terreno sobre el cual Manini volcó todo su pasado escenográfico, subvirtiendo y modificando el paisaje sintrense, pero también introduciendo nuevas tipologías arquitectónicas hasta entonces denostadas. Tipologías que, andando el tiempo, habrían de convertirse en referente fundamental de su ideal de belleza, cimentado, como sabemos, en base a un acusado gusto arqueológico-historicista y a una refinadísima concepción paisajística de lo pintoresco.

A través de este breve recorrido, se han puesto de relieve los principales logros alcanzados por Manini en el campo de la arquitectura y del paisaje, inmortalizado en sus cuatro obras señeras. La introducción de tipologías centroeuropeas (Biester), tomadas de las viejas villas de Hugo Licht, precedió a grandes propuestas como la Villa Sassetti de su coetáneo Sebastiano Locati. Villa Sassetti introdujo el estilo lombardo de los montes brescianos pero, sobre todo, sometió la arquitectura a extremos de una irresistible visualidad a través de una premeditada y cuidada búsqueda de la potencialidad 
del lugar. Chalet Lima Mayer obró el milagro: sus diseños escenográficos se tornaron en arquitectura, convirtiendo a Sintra en el escenario de un auténtico Tannhäuser. ¿Qué decir de la Quinta da Regaleira, su obra más aclamada y bandera izada del lusismo neomanuelino, hasta el punto de ser recordado más por ello que por las consabidas conquistas artísticas precedentes?

Manini y Sintra, Sintra y Manini constituyen, actualmente, un binomio artístico difícilmente separable. Emulando a los grandes artistas renacentistas, Manini dispuso de una ciudad para convertirla y predefinirla en arte, imprimiendo en ella su ideal de belleza así como su concepción de ciudad romántica. Una vez concluida su gran obra, recogió sus aperos $y$, silenciosamente, se retiró a la sierra bresciana -de extraña concomitancia sintrense- donde, cuentan, siguió evocando el Monte da Lúa hasta el final de sus días.

\section{Bibliografía}

ALPINI, Cesare (2007): "Manini Pittore", en Gaia Piccarolo y Giuliana Ricci (coords.), Luigi Manini (1848-1936) architetto e scenografo pittore e fotografo, pp. 117-129. Catálogo de la exposición celebrada en Crema, Citadella della Cultura, del 6 de mayo al 8 de julio de 2007. Milano: Silvana Editoriale.

ATTLEE, Helena (2007): Gardens of Portugal. London: Frances Lincoln.

CUNHA, Joao (2004): "Chalet Biester", en Luis Trigueiros (ed.), José Luis Monteiro (1848-1942), pp. 59-71. Lisboa: Blau.

D'AMIA, Giovanna (2007): "Luigi Manini scenografo. Dalla formatione scaligera ai trionfi portoghesi", en Gaia Piccarolo y Giuliana Ricci (coords.), Luigi Manini (1848-1936) architetto e scenografo pittore e fotografo, pp. 53-65. Catálogo de la exposición celebrada en Crema, Citadella della Cultura, del 6 de mayo al 8 de julio de 2007. Milano: Silvana Editoriale.

DENBY, Elaine (1998): Grand Hotels: Reality and Illusion; an architectural and Social History. London: Reaktion Books.

FERREIRA, Teresa (2007): "Scambi culturali tra l'Italia e il Portogallo, attività di Alfredo d'Andrade e Sebastiano Guiuseppe Locati in Portogallo", en Gaia Piccarolo y Giuliana Ricci (coords.), Luigi Manini (1848-1936) architetto e scenografo pittore e fotografo, pp. 65-73. Catálogo de la exposición celebrada en Crema, Citadella della Cultura, del 6 de mayo al 8 de julio de 2007. Milano: Silvana Editoriale.

LUCKHURST, Gerald; y PEREIRA, Denise (2004): "Luigi Manini no Buçaco". Monumentos, vol. 20, pp. 51-63.

LUCKHURST, Gerald; y PEREIRA, Denise (2007): "Dalla poetica della natura alla costruzione del paesaggio e del giardino. L'interpretazione di Luigi Manini", en Gaia Piccarolo y Giuliana Ricci (coords.), Luigi Manini (1848-1936) architetto e scenografo pittore e fotografo, pp. 87-99. Catálogo de la exposición celebrada en Crema, Citadella della Cultura, del 6 de mayo al 8 de julio de 2007. Milano: Silvana Editoriale.

MALCOLM, Jack (2003): Sintra, a Glorius Eden. London: Carcanet Press Ltd. 
PEREIRA, Denise (2012): "A cenografia oitocentista como fonte de cultura visual e de ideologia". Historia da Arte, vol. 10, pp. 147-159.

PEREIRA, Denise; y LUCKHURST, Gerald (2004): "A Vila Sassetti, projecto do cenógrafo-arquitecto Luigi Manini". Varia Escrita, vol. 11, pp. 253280.

PEREIRA, Denise; y LUCKHURST, Gerald (2006a): "Luigi Manini: esboço de uma biografia", en J. Cruz Alves (coord.), Luigi Manini: Imaginário y Método, Arquitectura y Cenografia, pp. 11-19. Catálogo de la exposición celebrada en Sintra, del 29 de Junio al 31 de octubre de 2006. Sintra: Cultur-Sintra.

PEREIRA, Denise; y LUCKHURST, Gerald (2006b): "Cenografia e espectáculo", en J. Cruz Alves (coord.), Luigi Manini: Imaginário y Método, Arquitectura y Cenografia, pp. 21-63. Cátalogo de la exposición celebrada en Sintra, del 29 de junio al 31 de octubre de 2006. Sintra: CulturSintra.

PEREIRA, Denise; y LUCKHURST, Gerald (2007): "Luigi Manini e I'architettura aulica in Portogallo", en Gaia Piccarolo y Giuliana Ricci (coords.), Luigi Manini (1848-1936) architetto e scenografo pittore e fotografo, pp. 73-87. Catálogo de la exposición celebrada en Crema, Citadella della Cultura, del 6 de mayo al 8 de julio de 2007. Milano: Silvana Editoriale.

PEREIRA, Denise; y LUCKHURST, Gerald (2008): "Luigi Manini e o projecto da Vila Sassetti". Monumentos, vol. 26, pp. 200-210.

PICCAROLO, Gaia (2005): Luigi Manini (1848-1936). L'opera architettonica di uno scenografo italiano in Portogallo. Tesis de Licenciatura no publicada. Politécnico de Milán.

PICCAROLO, Gaia (2007): "Villa e Villino: il tema dell'abitazione Borghese nell'opera di Luigi Manini", en Gaia Piccarolo y Giuliana Ricci (coords.), Luigi Manini (1848-1936) architetto e scenografo pittore e fotografo, pp. 99-109. Catálogo de la exposición celebrada en Crema, Citadella della Cultura, del 6 de mayo al 8 de julio de 2007. Milano: Silvana Editoriale.

RONCAI, Luciano (2007): "Giardini e ville tra Ottocento e Novecento nel cremonese", en Gaia Piccarolo y Giuliana Ricci (coords.), Luigi Manini (1848-1936) architetto e scenografo pittore e fotografo, pp. 39-47. Catálogo de la exposición celebrada en Crema, Citadella della Cultura, del 6 de mayo al 8 de julio de 2007. Milano: Silvana Editoriale. 